\title{
Associative formal power series in two indeterminates
}

Jörg Tomaschek ${ }^{\star}$ (University of Luxembourg)

Harald Fripertinger (Karl-Franzens-Universität Graz)

Ludwig Reich (Karl-Franzens-Universität Graz)

Jens Schwaiger (Karl-Franzens-Universität Graz)

The classical examples of associative formal power series are formal group laws. A one dimensional formal group law over a ring $\mathbb{A}$ is a formal power series $F, F \in$ $\mathbb{A}[[X, Y]]$ in two indeterminates of order one, such that

$$
F(X, Y)=X+Y+\sum_{\alpha+\beta \geq 2} a_{\alpha, \beta} X^{\alpha} Y^{\beta}
$$

and the associativity equation

$$
F(F(X, Y), Z)=F(X, F(Y, Z))
$$

holds, see [2]. One dimensional formal group laws as well as associative polynomials, see [3], and associative rational functions, see [1], are completely described.

For a commutative field $\mathbb{K}$ we characterize all formal power series $F \in \mathbb{K}[[X, Y]]$ of arbitrary order which are associative.

[1] A. Chéritat, Fractions rationelles associatives et corps quadratiques, Revue des Mathématiques de l'Enseignement Superieur, 109 (1998-1999) 1025-1040.

[2] M. Hazewinkel, Formal Groups and Applications, Acad. Press, New York and London, 1978.

[3] J.-L. Marichal, P. Mathonet, A description of n-ary semigroups polynomial-derived from integral domains, Semigroup Forum 83(2) (2011) 241-249. 